University of Nebraska - Lincoln

DigitalCommons@University of Nebraska - Lincoln

Faculty Publications, Department of Physics and Astronomy

Research Papers in Physics and Astronomy

$10-1-2000$

\title{
Magnetic properties of Ni nanowires in self-assembled arrays
}

\author{
M. Zheng \\ University of Nebraska-Lincoln \\ L. Menon \\ University of Nebraska-Lincoln \\ H. Zeng \\ University of Nebraska-Lincoln
}

Yi Liu

University of Nebraska-Lincoln, yliu@unl.edu

Supriyo Bandyopadhyay

University of Nebraska-Lincoln, sbandy@vcu.edu

See next page for additional authors

Follow this and additional works at: https://digitalcommons.unl.edu/physicsfacpub

Part of the Physics Commons

Zheng, M.; Menon, L.; Zeng, H.; Liu, Yi; Bandyopadhyay, Supriyo; Kirby, Roger D.; and Sellmyer, David J., "Magnetic properties of Ni nanowires in self-assembled arrays" (2000). Faculty Publications, Department of Physics and Astronomy. 40.

https://digitalcommons.unl.edu/physicsfacpub/40

This Article is brought to you for free and open access by the Research Papers in Physics and Astronomy at DigitalCommons@University of Nebraska - Lincoln. It has been accepted for inclusion in Faculty Publications, Department of Physics and Astronomy by an authorized administrator of DigitalCommons@University of Nebraska Lincoln. 


\section{Authors}

M. Zheng, L. Menon, H. Zeng, Yi Liu, Supriyo Bandyopadhyay, Roger D. Kirby, and David J. Sellmyer 


\title{
Magnetic properties of Ni nanowires in self-assembled arrays
}

\author{
M. Zheng, ${ }^{1,4}$ L. Menon, ${ }^{2,4}$ H. Zeng, ${ }^{1,4}$ Y. Liu, ${ }^{3,4}$ S. Bandyopadhyay, ${ }^{2,4}$ R. D. Kirby, ${ }^{1,4}$ and D. J. Sellmyer ${ }^{1,4}$ \\ ${ }^{1}$ Department of Physics \& Astronomy, University of Nebraska, Lincoln, Nebraska 68588 \\ ${ }^{2}$ Department of Electrical Engineering, University of Nebraska, Lincoln, Nebraska 68588 \\ ${ }^{3}$ Department of Mechanical Engineering, University of Nebraska, Lincoln, Nebraska 68588 \\ ${ }^{4}$ Center for Materials Research and Analysis, University of Nebraska, Lincoln, Nebraska 68588
}

(Received 6 April 2000)

\begin{abstract}
Magnetic properties of Ni nanowires electrodeposited into self-assembled porous alumina arrays have been investigated. By anodizing aluminum in sulfuric acid and immersing the as-anodized template into phosphoric acid for different lengths of time, we are able to vary the diameters of the subsequently deposited nanowires between 8 and $25 \mathrm{~nm}$. The coercivity measured along wire axis first increases with the wire diameter, reaches a maximum of 950 Oe near a diameter of $18 \mathrm{~nm}$, and then decreases with further increase of wire diameter. The dependence of the magnetization of Ni nanowires is found to follow Bloch's law at low temperature but with the Bloch exponent decreasing from the bulk value and the Bloch constant increasing from the bulk value by an order of magnitude.
\end{abstract}

\section{INTRODUCTION}

There has been increasing interest in the fabrication of nanostructured magnetic materials because of their unusual properties compared to bulk materials. They may also have applications in magnetic recording media, sensors and other devices. ${ }^{1-3}$ Many different techniques have been employed to produce nanoscaled materials. Among them, electrodeposition of nanodots or nanowires into self-assembled alumites is a simple, low-cost and high throughput technique. ${ }^{4-6}$

When aluminum is anodized in an acidic electrolyte, it forms porous alumina membranes with excellent uniformity in diameter and spacing of the ordered hexagonal holes. ${ }^{7,8}$ The diameter and spacing can be controlled by the anodizing conditions such as the electrolyte selected, anodizing time, temperature, and voltage. For example, when anodizing $\mathrm{Al}$ in sulfuric acid, the diameter of the pores is about $8 \mathrm{~nm}$ with an interpore distance of around $35 \mathrm{~nm}$; the diameter increases to about $70 \mathrm{~nm}$ with spacing $110 \mathrm{~nm}$ when oxalic acid is employed. The diameter can also be increased by anodizing the $\mathrm{Al}$ in sulfuric acid and immersing the as-made template in phosphoric acid for different lengths of time. We call this process " with the wire diameter, length and wire separation. In this paper, we investigate the size effect on the magnetic properties of Ni nanowires deposited in alumites.

\section{EXPERIMENTAL METHODS}

A $99.997 \%$ purity $\mathrm{Al}$ sheet was used as a starting material. It was first electropolished in a mixed solution of $165 \mathrm{ml}$ $65 \% \mathrm{HClO}_{4}, 700 \mathrm{ml}$ ethanol, $100 \mathrm{ml}$ 2-butoxyethanol, and $137 \mathrm{ml} \mathrm{H}_{2} \mathrm{O}$. Then it was anodized in acidic solutions. Two different anodization procedures were used. To obtain highly ordered pores, a prolonged three-step anodization ${ }^{8}$ was employed. (1) Anodize Al at $40 \mathrm{~V}$ dc in $3 \%$ oxalic acid for about $10 \mathrm{~min}$ to form textures on $\mathrm{Al}$ surface and then remove the formed oxide layer by immersing anodized Al into a mixed solution of $0.2 \mathrm{M}$ chromic acid and $0.4 \mathrm{M}$ phosphoric acid for $15 \mathrm{~min}$. (2) Anodize under the same anodization condition for longer time, e.g., $12 \mathrm{~h}$ and remove the top oxide layer again in the same way mentioned above. (3) Perform a third three-minute anodization. Figure 1 shows an atomic force microscopy (AFM) top view of the template after this three-step anodization. Uniform pores with an average pore diameter of about $70 \mathrm{~nm}$ and center-to-center spacing of about $110 \mathrm{~nm}$ were achieved. To obtain smaller diameter and center-to-center spacing of the nanopores, a second procedure was employed where sulfuric acid was used as the anodization solution.

In this study, all the samples were first anodized in $15 \%$ sulfuric acid under constant voltage of $10 \mathrm{~V}$ for $30 \mathrm{~min}$. The pores of the anodic alumina were widened by immersing the as-made template in $1 \%$ phosphoric acid for different lengths of time. Ni was electrodeposited in the as-made or porewidened template using an electrolyte containing $0.1 \mathrm{M} \mathrm{NiSO}_{4} \cdot 6 \mathrm{H}_{2} \mathrm{O}$ and $45 \mathrm{~g} / \mathrm{L}$ boric acid. The electrodeposition was carried out at $55^{\circ} \mathrm{C}$ using an alternating current with a voltage of $15 \mathrm{~V}$ and frequency of $250 \mathrm{~Hz}$.

Transmission electron microscopy (TEM) and AFM were used to determine the topography, diameter, spacing, and length of the alumites pores. The TEM plan-view samples were prepared by standard dimpling and ion-milling processes. The nanowires were released for TEM study by submerging the specimen in a mixed solution of $0.2 \mathrm{M}$ chromic acid and $0.4 \mathrm{M}$ phosphoric acid and caught by carbon grid. Magnetic measurements were carried out with an alternatinggradient force magnetometer (AGFM). Low-temperature magnetization measurements were carried out using a superconducting quantum interference device (SQUID).

\section{RESULTS AND DISCUSSION}

\section{A. Morphology and structure}

When anodizing $\mathrm{Al}$ in $15 \%$ sulfuric acid, the diameter and spacing were about 8 and $35 \mathrm{~nm}$, respectively, and the pore density exceeds $10^{11} / \mathrm{cm}^{2}$. These features are much smaller than those obtained with oxalic-acid-anodized samples. After 


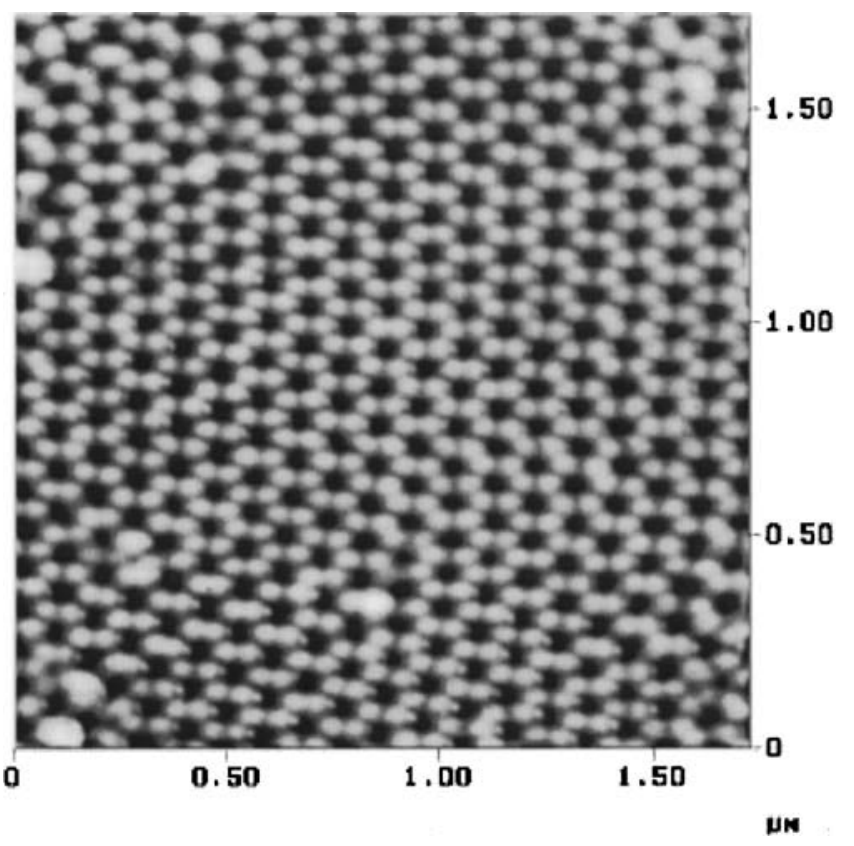

FIG. 1. AFM top view of a nanopore array in anodic alumina prepared by three-step anodization. Anodization was conducted in $3 \%$ oxalic acid at $40 \mathrm{~V}$.

anodization, the as-anodized sample was immersed into $1 \%$ phosphoric acid for different lengths of time to widen the pores without changing the center-to-center spacing. Figure 2(a) shows a plan-view TEM micrograph of the sample porewidened for $17 \mathrm{~min}$. The average center to center distance between adjacent pores is about $35 \mathrm{~nm}$. The darker contrast at the upper-left corner is due to the thickness of the speci-

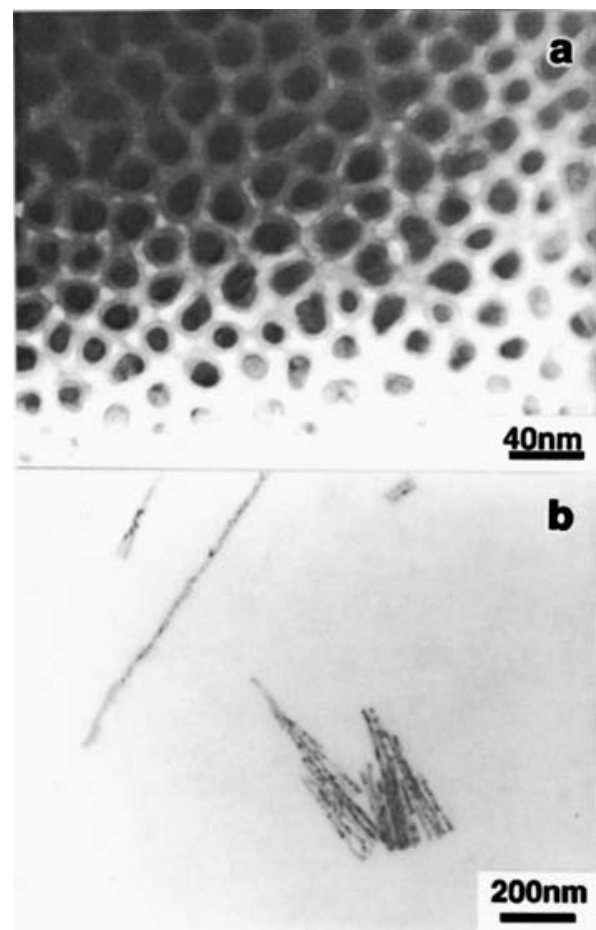

FIG. 2. (a) TEM plan-view of the sample anodized in $15 \%$ sulfuric acid for $30 \mathrm{~min}$ and followed by pore widening in $1 \%$ phosphoric acid for $17 \mathrm{~min}$. (b) TEM micrograph of Ni nanowires freed from the alumites.

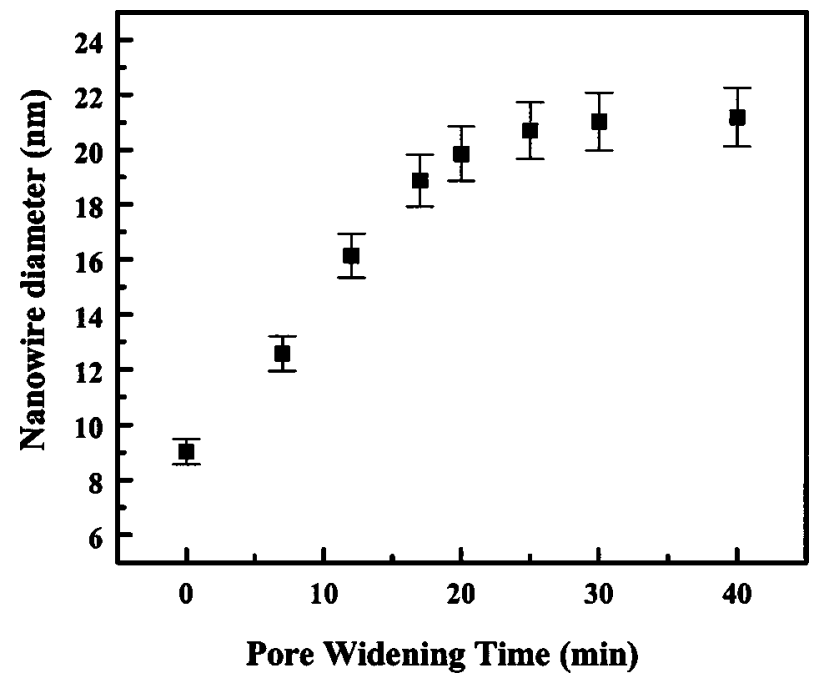

FIG. 3. The mean diameter of nanowires $d$ as a function of pore widening time.

men. Figure 2(b) shows the TEM image of Ni nanowires liberated from anodic films, revealing that the nanowires are cylindrical with lengths around $1 \mu \mathrm{m}$. Each wire is polycrystalline as revealed by high-resolution imaging and electron diffraction. Though the freed wires may be oxidized, we estimate the oxidized layer to be less than 1-2 monoatomic layers, which is $0.2-0.4 \mathrm{~nm}$. Considering that the smallest diameter of these nanowires is about $10 \mathrm{~nm}$, the error will be less than $4 \%$. So we can determine the diameters by TEM. The average diameter of the wires is plotted as a function of pore-widening time in Fig. 3. The diameter increases linearly with pore widening time for a short duration up to about 20 min and then the increase nearly saturates. This is because the maximum diameter is limited by the separation between two wires. Detailed TEM investigation will be reported in a forthcoming paper.

$\mathrm{X}$-ray diffraction (XRD) analysis cannot directly be used to determine the structure of the embedded Ni nanowires in alumina since some of the diffraction peaks of $\mathrm{Ni}$ are very close to those of $\mathrm{Al}$ and the intensities of the $\mathrm{Al}$ peaks from the substrate are much greater than those of Ni. Specimens were immersed in $3 \%$ mercuric chloride solution and allowed to stand for half an hour at room temperature to strip the oxide. The stripped oxide films were then washed, dried and measured by XRD. Figure 4 shows the typical XRD pattern of the Ni nanowires deposited in an as-made template $(d \sim 8 \mathrm{~nm})$ and those with pore widened for 17 and $30 \mathrm{~min}$ $(d \sim 18,21 \mathrm{~nm})$. The crystal structure of the Ni wires is fcc. From the figure, one can see that the half-width of the diffraction peak decreases with the pore widening time, suggesting an increase of grain size with pore widening. The grain sizes estimated from Scherrer's formula, which correspond to a coherence length along the wire axis, are 13, 18.6, and $24.8 \mathrm{~nm}$ for Ni nanowires with diameter of 8,18 , and 21 $\mathrm{nm}$, respectively. It is interesting that this coherence length scales approximately with the wire diameter.

\section{B. Magnetic reversal}

Thermal stability and time-dependent coercivity are important issues in magnetic nanostructures and their applica- 


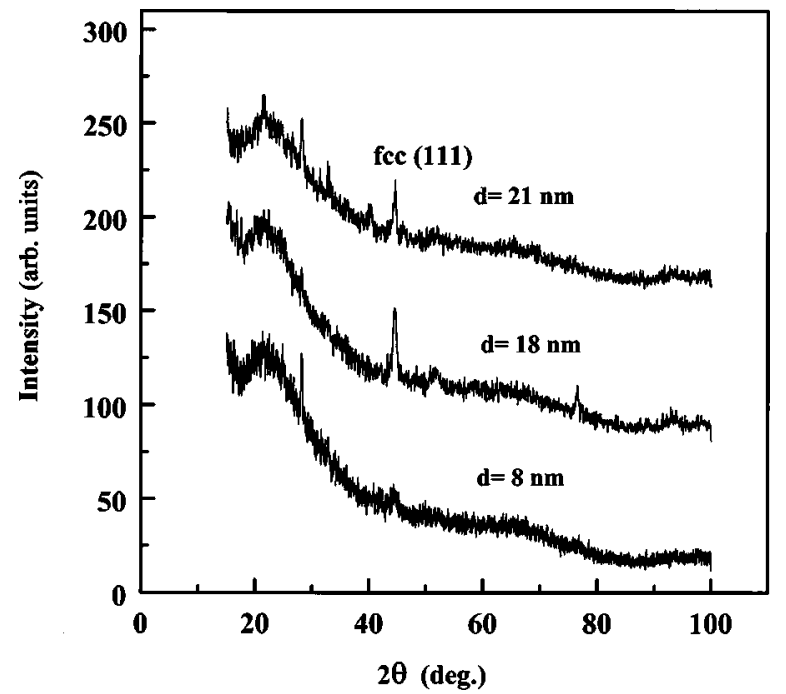

FIG. 4. XRD pattern of $\mathrm{Ni}$ nanowires in as-made and porewidened for 17 and 30 min templates. The corresponding diameter of nanowires for the three samples is $8,18,21 \mathrm{~nm}$ separately.

tions. Both effects are related to thermally activated magnetization processes. Magnetic viscosity $S$, activation volume $V^{*}$, and $\Delta M$ plots measurements are often used to determine thermal stability and magnetic interactions in nanoscaled particles and grains. ${ }^{9}$

Figure 5 gives the coercivity as a function of pore widening time, i.e., wire diameters. The coercivity is measured with the magnetic field applied along the wire axis. The corresponding hysteresis loops for Ni nanowires with diameters of 8,18 , and $21 \mathrm{~nm}$ are shown as insets. The Ni nanowires have easy axes along the wire axis, i.e., perpendicular to the film plane due to the shape anisotropy. For $\mathrm{Ni}$ nanowires deposited into an as-made template, the coercivity is about 480 Oe. The coercivity increases with pore-widening time and reaches the highest value, $950 \mathrm{Oe}$, for $\mathrm{Ni}$ deposited into a template which had been pore-widened for $17 \mathrm{~min}$ (wire diameter about $18 \mathrm{~nm}$ and spacing of $35 \mathrm{~nm}$ ). The coercivity then decreases with further increase of pore diameter. The remanence ratios $M_{r} / M_{s}$ for these three samples are 0.77 , 0.84 , and 0.33 , respectively.

The coercivity dependence of Ni nanowire diameters has been studied by other groups. ${ }^{10,11} \mathrm{Ni}$ nanowires with diameter between 30 to $200 \mathrm{~nm}$ were electrodeposited into random arrays of track-etched polycarbonate membranes. Whitney et al. ${ }^{10}$ found that the coercivities of $\mathrm{Ni}$ nanowires increase with decreasing wire diameter. They attributed to the multidomains formed for the larger-diameter wires which degrades the coercivity. The authors suggested that coercivity can be further enhanced by improving the process for fabricating wires with diameter less than $50 \mathrm{~nm}$, which has been proved by our results. Ferré et al. ${ }^{11}$ also found a similar coercivity dependence on wire diameters. They observed that though $H_{c}$ takes on values that are not far from those predicted by the curling or buckling nucleation mechanism in the infinite cylinder, the dependence of $H_{c}$ on wire diameter does not agree with any of the nucleation mechanisms proposed. Although the characteristic coercivity of nanowires is mainly determined by their diameters, it has been found that coercivity also depends on the packing density of the alumite

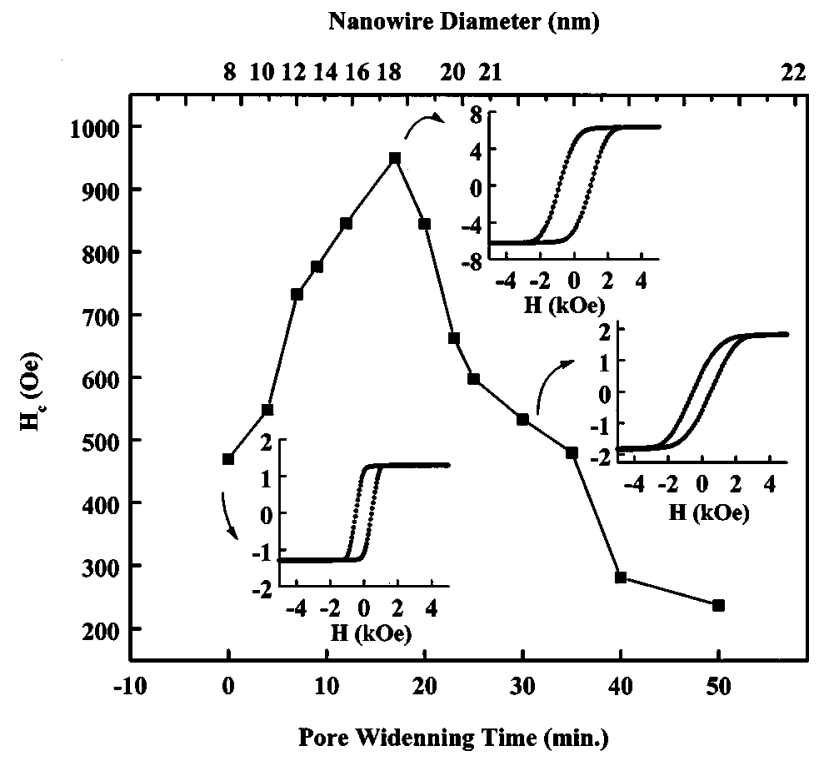

FIG. 5. Dependence of the coercivity vs the pore-widening time, i.e., nanowire diameter. The corresponding hysteresis loops for $\mathrm{Ni}$ nanowires with diameter of 8,18 , and $21 \mathrm{~nm}$ are shown as inset.

template. ${ }^{6,12}$ Coercivity of nanowires decreases slightly with increasing packing density, and this can be explained in terms of interwire magnetostatic interactions. ${ }^{6,12}$

Previous studies on magnetization reversal of nanowires treated the wires as single-domain infinite cylindrical particles. Two reversal modes have been suggested as being important, curling and the coherent rotation mode. ${ }^{12}$ There exists a critical radius $R_{c}$ : If $R<R_{c}$, reversal occurs by coherent-rotation, and if $R>R_{c}$, the reversal occurs by curling (see, e.g., Refs. 9, 13, and references therein). The critical radius $R_{c}$ is given by

$$
R_{c}=\left(\frac{k A}{\mu_{0} M_{s}^{2}}\right)^{1 / 2}
$$

where $k$ is a constant depending on the length/diameter ratio of the particles, ranging from 1.08 for an infinite cylinder to 1.38 for a sphere. $A$ is the exchange constant, 0.3 $\times 10^{-6} \mathrm{erg} / \mathrm{cm}$ for $\mathrm{Ni}$. Using the saturation magnetization value of bulk Ni, the calculated $R_{c}$ is about $13 \mathrm{~nm}$. For an ideal system, the variation of coercivity is relatively flat for diameters smaller than the critical diameter and it decreases for diameter larger than the critical diameter. In reality, because of defects and thermally activated processes, the variation of the coercivity usually shows an increase before decreasing rapidly when the particle radius crosses the critical radius. $^{13}$

Although the dependence of the coercivity on wire diameter can be qualitatively explained by the above reversal mechanism, Eq. (1) estimates the critical radius to be $13 \mathrm{~nm}$, while our data suggest that $R_{c} \approx 9 \mathrm{~nm}$. In particular, the maximum coercivity in Fig. 5 is about three times smaller than the predicted value from coherent rotation. Recent theoretical work ${ }^{14}$ and experimental results ${ }^{15}$ showed that, when the crystalline anisotropy is small and the dipolar contribution dominates, the switching filed could be less than the switching filed for coherent rotation, by a factor of 3. Recently Skomski et al. suggested that the onset of magnetiza- 


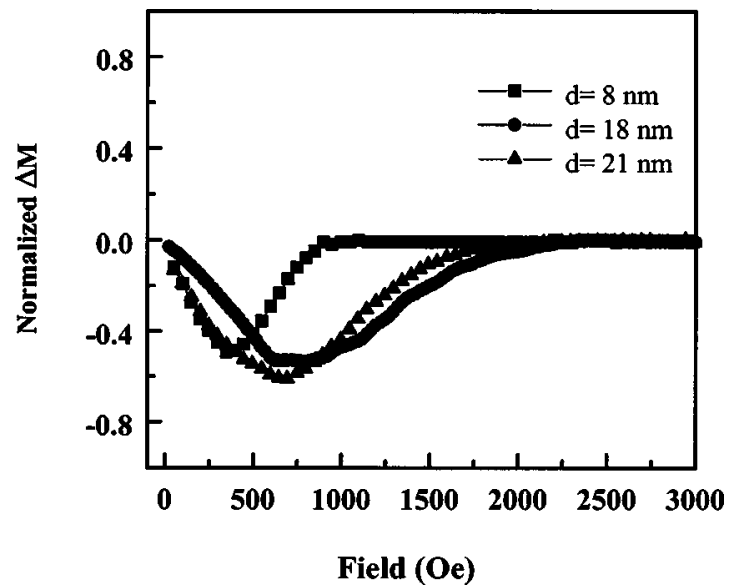

FIG. 6. Three representative $\Delta M$ plots for Ni nanowires with diameter of 8,18 , and $21 \mathrm{~nm}$.

tion reversal is localized, as opposed to the assumed delocalized coherent rotation and curling modes. ${ }^{16}$ The localization caused by morphological inhomogenities is largely responsible for the observed hysteretic behavior. Polycrystalline Ni nanowires can be treated as random-anisotropy ferromagnets where the random-anisotropy forces associated with the local uniaxial anisotropy compete with the interatomic exchange and external fields, which favor magnetization alignment. As a result, the magnetization processes become localized. Quantitative theoretical calculations, which involve scaling law and crossover, are under way and will be published elsewhere.

$\Delta M$ plots, ${ }^{17}$ derived from dc demagnetization remanence and isothermal remanence curves, show negative peaks for all the samples investigated. This reveals that a predominant interaction for our samples is magnetostatic. Figure 6 gives three representative $\Delta M$ plots for $\mathrm{Ni}$ nanowires with diameter of 8,18 , and $21 \mathrm{~nm}$, respectively.

\section{Temperature dependence}

Figure 7(a) shows the low-temperature magnetization behavior for Ni nanowires with diameter of about $8 \mathrm{~nm}$ and length of about $1.4 \mu \mathrm{m}$. The magnetization was measured with a SQUID at 5000 Oe to ensure that the magnetization was saturated. An alumite template prepared under the same anodization conditions has been measured to subtract the background signal, which was about two orders of magnitude smaller than that due to the Ni nanowires. The magnetization for bulk $\mathrm{Ni}$ is shown for comparison.

Aside from anisotropy effects, the spontaneous magnetization of bulk magnetic materials at low temperature is determined by spin-wave and follows the Bloch law ${ }^{18}$

$$
M(T)=M(0)\left(1-B T^{\lambda}\right),
$$

where $B$ is the Bloch constant and $\lambda=\frac{3}{2}$, is the threedimensional Bloch exponent.

Previous investigations of low-temperature behavior of nanoparticles showed mixed results. Mills and Maradudin considered the surface and calculated the spin-wave contri-

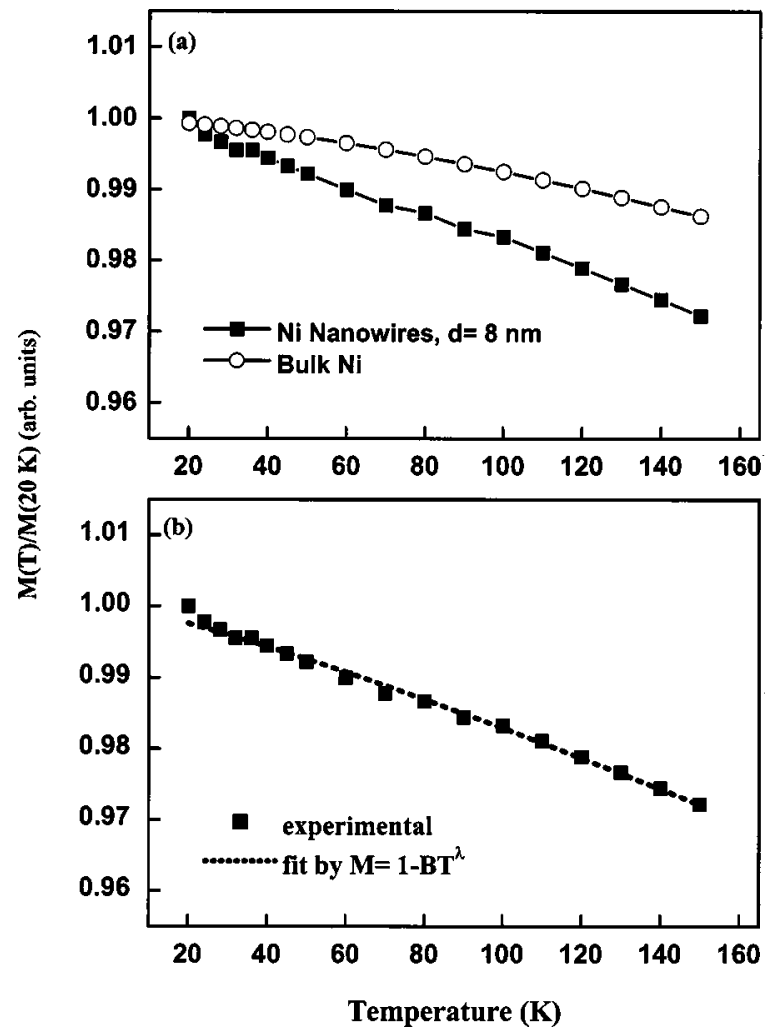

FIG. 7. (a) The low-temperature magnetization dependence of $\mathrm{Ni}$ nanowires, where the behavior of bulk $\mathrm{Ni}$ is shown as comparison. The measurement was conducted by SQUID at a field of 5000 Oe. (b) The experimental curve and fitting result using $M(T) / M(20 k)=1-B T^{\lambda}$ of Ni nanowires, the fitted $B$ and $\lambda$ are $B=6 \times 10^{5}$ and $\lambda=1.21 \pm 0.04$, respectively.

bution of the "nonbulk" system and found the Bloch exponent remained $\lambda=\frac{3}{2}$ as in the bulk, but the Bloch constant of the surface was predicted to be twice that of the bulk, $B_{\text {surf }}$ $=2 B_{\text {bulk }} \cdot{ }^{19}$ Xiao and Chien obtained $\lambda=\frac{3}{2}$ with $B_{\text {surf }}$ $=10 B_{\text {bulk }}$ for $2 \mathrm{~nm}$ Fe nanoparticles embedded in $\mathrm{SiO}_{2}$ at $50 \%$ volume fraction. ${ }^{20}$ Recently, Zhang et al. ${ }^{21}$ found that Fe nanoscaled particles obey the form of Bloch's law as written above. However, with size decreasing from 18 to 3 $\mathrm{nm}$, the Bloch exponent falls from the bulk value to smaller values at diameter of about $6 \mathrm{~nm}$ and the Bloch constant rises from the bulk value by an order of magnitude.

Using Eq. (2), we fitted our results for Ni nanowires. The fitted results are shown in Fig. 7(b). We obtained $\lambda=1.21$ \pm 0.04 and $B=6 \times 10^{-5}$ which is about an order bigger than the bulk value. Our results are quite consistent with Ref. 20. One possible explanation ${ }^{21}$ is that the reduced coordination at the surface causes the spins at the surface to be more susceptible to thermal excitation which leads to larger temperature dependence of the magnetization. Note, however, that the three-dimensional integration yielding Bloch's law breaks down for very small wave vectors. This regime, where all spin waves are parallel to the wire axes yields deviations from Bloch's law at extreme low temperatures (Fig. 7).

In summary, the coercivity of $\mathrm{Ni}$ nanowires was found to depend on wire diameter, increasing from 480 Oe at diameter of $8 \mathrm{~nm}$ to 950 Oe near $18 \mathrm{~nm}$ and then decreasing with 
further increasing wire diameter. At low temperature, magnetization of Ni nanowires can be described by Bloch's law with a higher stiffness constant and a lower exponent than that of the bulk Ni. We ascribe these phenomena to the reduced dimensionality and the surface effects in the $\mathrm{Ni}$ nanowires.

\section{ACKNOWLEDGMENTS}

The authors would like to thank R. Skomski and P. A. Dowben for useful discussions. The work was supported by NSF, ARO, and CMRA. H. Zeng was supported by IBM.
${ }^{1}$ Nanophase Materials, edited by G. C. Hadjipanayis and R. W. Siegel (Kluwer, Dordrecht, 1994).

${ }^{2}$ K. Liu, K. Nagodawithana, P. C. Searson, and C. L. Chien, Phys. Rev. B 51, 7381 (1995).

${ }^{3}$ D. J. Sellmyer, M. Yu, R. A. Thomas, Y. Liu, and R. D. Kirby, Phys. Low-Dimens. Semicond. Struct. 1/2, 155 (1998).

${ }^{4}$ D. AlMawlawi, N. Coombs, and M. Moskovits, J. Appl. Phys. 70, 4421 (1991).

${ }^{5}$ R. M. Metzger, V. V. Konovalov, M. Sun, T. Xu, G. Zangari, B. Xu, M. Benakli, and W. D. Doyle, IEEE Trans. Magn. 36, 30 (2000)

${ }^{6}$ H. Zeng, M. Zheng, R. Skomski, D. J. Sellmyer, Y. Liu, L. Menon, and S. Bandyopadhyay, J. Appl. Phys. 87, 4718 (2000).

${ }^{7}$ F. Keller, M. S. Hunter, and D. L. Robinson, J. Electrochem. Soc. 100, 411 (1953).

${ }^{8}$ H. Masuda and K. Fukuda, Science 268, 1466 (1995); H. Masuda, H. Yamada, M. Satoh, H. Asoh, M. Nakao, and T. Tamamura, Appl. Phys. Lett. 71, 2770 (1997).

${ }^{9}$ R. W. Chantrell, J. Magn. Magn. Mater. 95, 365 (1991).

${ }^{10}$ T. M. Whitney, J. S. Jiang, P. C. Searson, and C. L. Chien, Science 261, 1316 (1993).
${ }^{11}$ R. Ferré, K. Ounadjela, J. M. George, L. Piraux, and S. Dubois, Phys. Rev. B 56, 14066 (1997).

${ }^{12}$ Li Cheng-Zhang and J. C. Lodder, J. Magn. Magn. Mater. 88, 236 (1990); G. T. A. Huysmans and J. C. Lodder, J. Appl. Phys. 64, 2016 (1988).

${ }^{13}$ R. Skomski and J. M. D. Coey, Permanent Magnetism (Institute of Physics Publishing, Bristol, 1999).

${ }^{14}$ S. T. Chui and De-Cheng Tian, J. Appl. Phys. 78, 3965 (1995).

${ }^{15}$ A. Kent, S. von Molner, S. Goder, and D. D. Awschalom, J. Appl. Phys. 76, 6656 (1994).

${ }^{16}$ R. Skomski, H. Zeng, M. Zheng, and D. J. Sellmyer, Phys. Rev. B 62, 3900 (2000).

${ }^{17}$ G. W. D. Spratt, P. R. Bissell, R. W. Chantrell, and E. P. Wohlfarth, J. Magn. Magn. Mater. 75, 919 (1988).

${ }^{18}$ S. V. Vonsovskii, Magnetism (Wiley, New York, 1974).

${ }^{19}$ D. L. Mills and A. A. Maradudin, J. Phys. Chem. Solids 28, 1855 (1967); D. L. Mills, Solid State Phys. 4, 95 (1972).

${ }^{20}$ G. Xiao and C. L. Chien, J. Appl. Phys. 61, 3308 (1987).

${ }^{21}$ Dajie Zhang, K. J. Klaubunde, C. M. Sorensen, and G. C. Hadjipanayis, Phys. Rev. B 58, 14167 (1998). 